\title{
Short-term Progressive Resistance Training Increases Strength and Lean Body Mass in Adults Infected with Human Immunodeficiency Virus
}

Ronenn Roubenoff, Ann McDermott, Lauren Weiss, Juliet Suri, Michael Wood, Rina Bloch and Sherwood Gorbach

\begin{abstract}
Background: To assess the efficacy of progressive resistance training (PRT) in increasing strength and lean body mass (LBM) in HIV-infected adults.

Methods: Twenty-five adults with HIV infection were trained using a highly intensive PRT regimen for 8 weeks, followed by an additional 8 weeks of observation under ad libitum physical activity conditions.
\end{abstract}

Results: Twenty-four of the 25 patients completed the first phase of the study. They had significant increases in strength on all four exercises tested ( $P<0.0001)$, and an increase in LBM of $1.75 \pm 1.94$ $\mathrm{kg}$ (mean $\pm \mathrm{SD}, \mathrm{P}<0.0002)$, with a concomitant decline in fat of $0.92 \pm 2.22 \mathrm{~kg}(\mathrm{P}<0.05)$, and no significant change in weight or bone mineral content. Twenty-one of the patients returned for follow-up 8 weeks after completing the PRT. Compared with their baseline values, their mean lean mass remained $1.40 \pm 1.8 \mathrm{~kg}$ higher $(\mathrm{P}<0.003)$. Among those who continued to train to some extent, lean mass increased by a mean of $1.1 \pm 1.6 \mathrm{~kg}(\mathrm{n}=9, \mathrm{P}<0.05$ versus end of PRT), whereas those who did no further training showed an increase in lean mass of $0.28 \pm 1.4 \mathrm{~kg}(\mathrm{n}=12, \mathrm{P}=\mathrm{NS}$ versus end of PRT). The difference between the two groups was not, however, significant $(\mathrm{P}=0.25)$. Among six patients with AIDS wasting, the increase in LBM was larger than among non-wasted patients (2.8 versus $1.4 \mathrm{~kg}, \mathrm{P}<0.06)$, and there was an increase in both weight $(+3.9$ versus $-0.2 \mathrm{~kg}$, $\mathrm{P}<0.002)$ and fat mass ( 0.95 versus $-1.5 \mathrm{~kg}, \mathrm{P}<0.002)$ at 8 weeks, which persisted at 16 weeks (weight: +4.0 versus $-1.6 \mathrm{~kg}, \mathrm{P}<0.0002$; fat: +1.6 versus $-1.9 \mathrm{~kg}, \mathrm{P}<0.01$ ).

Conclusion: This preliminary study suggests that short-term, high intensity PRT can significantly increase LBM and strength in HIV infection, and may be used as an alternative or adjunct to pharmacological anabolic treatments in this disease. 


\section{Introduction}

Unintentional loss of weight in patients with HIV infection, or AIDS wasting, is a common complication of advanced disease, and causes weakness, disability, and death [1-6]. The most important effect of wasting is the loss of lean body mass (LBM), and especially of muscle mass, because muscle mass is a major determinant of strength and thus of functional capacity and disability [7]. Even in the absence of weight loss, lean mass and muscle mass can still be reduced, because there may be an increase in another component of body composition, such as extracellular water or fat. We have previously suggested that the term cachexia be reserved for this situation, in contrast to wasting [8]. Cachexia has similar effects on function and strength to those seen in wasting, although often to a milder degree.

Even with the advent of highly active antiretroviral therapy (HAART) for HIV infection, data from longitudinal studies at our center and elsewhere $[9,10]$ suggest that although weight gain occurs with HAART, a high proportion of this gain is in the fat compartment. Several anabolic treatments have been shown to increase LBM in HIV infection, including growth hormone, androgen therapy, and to a lesser extent megestrol acetate [11-14]. Growth hormone is very expensive, however, and may cause carpal tunnel syndrome, whereas androgens are associated with side-effects such as liver disorders, masculinization in women, and testicular atrophy, and perhaps an increased risk of prostate cancer in men $[15,16]$.

The only physiological intervention known to increase lean mass, muscle mass and strength is progressive resistance training (PRT). PRT has been shown to be effective in a variety of situations, including normal ageing, age-related frailty, and arthritis [17-19]. In each case, the requirement for a successful increase in lean mass has been the use of a highly intensive regimen $(75-80 \%$ of the one-repetition maximum (1-RM), the most weight a person can lift in good form). In addition, the regimen must be progressive: as people become stronger the 1-RM must be remeasured and the work load increased to maintain the high intensity. Although anecdotal reports suggest that many patients with HIV infection do strength train, there has, however, been no systematic study of highly intensive PRT to increase lean mass in HIV-infected adults. We now report on the results of an 8 week, highly intensive PRT intervention followed by 8 weeks of self-selected physical activity in patients with HIV infection, with or without AIDS wasting.

\section{Methods}

\section{Patient population}

Participants were recruited from the Tufts Nutrition for Life cohort, an ongoing study of over 575 men and women with HIV infection, who are being followed at 6 month intervals for an assessment of the effect of nutritional status on HIV disease progression. Inclusion criteria for the present study were interest in the study and the ability to give informed consent. AIDS wasting was not required for entry into the study, but six of the subjects met the criteria for wasting, defined as body mass index (BMI) of less than $20 \mathrm{~kg} / \mathrm{m}^{2}$ or an unintentional weight loss of $10 \%$ or more of their usual weight over the previous year [2]. Exclusion criteria included participation in resistance training over the 6 months preceding study entry, a contraindication to progressive resistance exercise 
(myocardial infarction within 6 months, unstable angina, uncontrolled congestive heart failure or uncontrolled hypertension), diabetes mellitus (an exclusion from the main cohort), renal insufficiency (serum creatinine $>2.0 \mathrm{mg} / \mathrm{dl}$ ), hepatic disease (aspartate aminotransferase (AST) or alanine aminotransferase (ALT) over five times the upper limit of normal, total bilirubin $>2.0$ $\mathrm{mg} / \mathrm{dl}$ ), muscle disease (creatine kinase $>500 \mathrm{IU} / \mathrm{ml}$ ), and arthritis or low back pain, limiting the activities of daily living. Because PRT has been shown to be useful in some or all of these conditions, we excluded them in order to study patients with HIV infection alone. Patients taking anabolic therapy (growth hormone, androgens, or megestrol acetate) were also excluded.

\section{Body composition and nutritional assessment}

Subjects were evaluated in the General Clinical Research Center (GCRC) at New England Medical Center. The usual dietary intake was available on all subjects from their participation in the Tufts Nutrition for Life cohort, based on food records kept on three non-consecutive days when the subjects were consuming a self-selected diet at home. The food records were collected at baseline, week 8, and week 16 of the study. A research registered dietitian taught the participants how to keep a 3 day food record using a food scale (Sunbeam Corporation, Mississauga, Ontario, Canada) and ruler. Food records were reviewed by the dietitian with the participant at each evaluation. The food records were evaluated using the Minnesota Nutrient Database, version 2.91. Details of the dietary intake of the main Nutrition for Life cohort have been submitted for publication elsewhere (Woods MN, Knox T, Spiegelman D, et al., unpublished observations).

LBM (fat-free, mineral-free mass), bone mineral content, and fat mass were estimated by dual energy X-ray absorptiometry (DXA) of the whole body using a Hologic QDR 2000 instrument operating in the array mode. We have previously demonstrated that this method gives estimates of fat and fat-free mass that are very close to those obtained with in-vivo neutron activation analysis, with a correlation of $r=0.87$ and a mean difference of less than $0.5 \mathrm{~kg}$ [20]. The coefficient of variation for five healthy persons measured twice over 2 days was $0.4 \%$ of total LBM and $1.4 \%$ for fat mass [21].

\section{Progressive resistance training protocol and strength assessment}

Each participant trained three times per week for 8 weeks using Keiser pneumatic resistance equipment (Keiser Sports Health Equipment Inc., Fresno, CA, USA). The double leg press, leg (quadriceps) extension, seated chest press, and seated row machines were used in order to focus on large muscle groups, which have an effect both on whole-body composition and on function. One subject had moderate chronic low back pain and did not perform the leg press or quadriceps extension exercises in most of his sessions. Baseline strength was assessed using the one-repetition maximum (1-RM) test, a measure of the most weight an individual can lift once with good form. The 1-RM was determined for each of the four exercise machines twice at baseline, and repeated every 2 weeks. Participants trained at $50 \%$ of their 1 -RM for the first session, $60 \%$ for the second session, and at $75-80 \%$ of their 1-RM for the remainder of the sessions. They performed three sets of eight repetitions on each machine, working with an exercise physiologist on a one-to-one basis or in small groups (two to three volunteers at a time). They were asked to rate the difficulty of each exercise at the end of each set using a Borg Relative Perceived Effort (RPE) Scale [22], and if the RPE was less than 16 out of a possible 20, the resistance was increased by $12 \mathrm{lbs}$ without waiting 
for the next scheduled 1-RM test. After each 1-RM test, the level of resistance was reset to $80 \%$ of the new (and higher) 1-RM, so that the training remained intensive and progressive during the entire 8 week program. We have previously used this approach successfully in our laboratory in frail elderly individuals and patients with rheumatoid arthritis, and observed large increases in strength without injury or dropout [18,19].

\section{Self-selected activity phase}

After the initial 8 week period, subjects were again evaluated in the GCRC as described above. Upon completing this evaluation, they were asked to return to their usual activity pattern. We did not think it ethical to discourage subjects who wished to continue to exercise from doing so, therefore we did not discourage this but asked all subjects to keep logs of their activity. The goal of this phase was to examine the change in strength and body composition during a period of time without investigator-initiated intervention. Therefore, during this phase subjects were not trained at our center, nor were they in contact with their trainers from the first phase. On the basis of self-report, nine of the participants continued to train at least once per week during the next 8 weeks, whereas 12 did no formal training. At the end of 8 weeks, they were again invited to the GCRC for evaluation.

\section{Statistical analysis}

All data were examined for normality graphically and statistically. The primary outcome of the study was the change in LBM assessed by DXA at 8 weeks and at 16 weeks after entry into the study. Secondary outcomes were changes in strength on each exercise during those periods. These were compared using analyses of variance (ANOVA). In addition, the differences in the primary and secondary outcomes by sex, race, medication use, wasting status, and continuation of training during the second 8 week study period were compared with baseline and week 8 using ANOVA. The relationship between change in lean mass or strength and dietary energy and protein intake was examined using linear regression. Differences between the groups were considered to be statistically significant if the two-tailed $P$-value was less than 0.05 .

\section{Results}

\section{Study population}

The study participants were 20 men and five women, with a mean age of 39 (range 25-56 years) and a mean BMI of $24.2 \mathrm{~kg} / \mathrm{m}^{2}$ (see Table 1). The study group included 11 African Americans and 14 whites. Nine of the volunteers had contracted HIV through intravenous drug use, 15 through homosexual contact, and one through a blood transfusion. The study was carried out between January, 1995 and May, 1998. Seven of the participants (28\%) were homeless or living in shelters. Six of the patients had AIDS wasting, defined as a loss of more than $10 \%$ of their usual body weight over the previous year or a BMI $\left(\mathrm{kg} / \mathrm{m}^{2}\right)$ below $20 \mathrm{~kg} / \mathrm{m}^{2}$ [2]. At the start of the study no subjects were participating in regular exercise, defined as $30 \mathrm{~min}$ or more of exercise that leads to perspiration at least three times a week, but three subjects had had experience with weight-lifting in the past. None of these three had been training in the previous 6 months. Mean $( \pm S D)$ dietary 
energy intake was $2592 \pm 723 \mathrm{kcal} /$ day, and did not differ significantly between wasted $(2842 \pm$ $363 \mathrm{kcal} /$ day) and non-wasted subjects (2532 $\pm 198 \mathrm{kcal} /$ day, $P=0.71)$.

Two participants were taking no antiretroviral therapy, nine were taking monotherapy or dual therapy (generally zidovudine (AZT) and lamivudine), and 14 were taking highly active antiretroviral therapy, defined as taking two protease inhibitors or three or more drugs, in which at least one was a protease inhibitor or a non-nucleoside reverse transcriptase inhibitor. Subjects were asked not to change their medication regimen during the study unless it was medically necessary. No medication changes occurred during the first 8 week phase, but two patients began protease inhibitors during the second 8 week phase. The median serum HIV RNA level was 8918 copies/ml (range undetectable to 45899 copies $/ \mathrm{ml}$ ), and did not change significantly over the 16 weeks of follow-up (Table 1). Mean CD4 count was 328 cells/ml (range 38-681 cells/ml, and did not change significantly during the study (Table 1 ).

Of the 25 individuals who enrolled in the study, 24 (96\%) completed the first 8 week phase, and 21 (84\%) completed the second 8 week phase. Adherence with the exercise regimen was excellent, with all 24 participants who completed phase 1 having a $90 \%$ or better attendance rate. One subject dropped out in week 6 of the first phase because of scheduling problems. Three other men were lost to follow-up during the second phase: one left town because of an outstanding warrant for his arrest, and two homeless men who could not be located within one month of their scheduled week 16 visit were excluded. The response to exercise in the dropouts was not significantly different from those who continued the study in terms of strength change or change in body composition.

\section{Effect of progressive resistance training on strength and body composition}

After 8 weeks of PRT, the mean 1-RM increased by $31 \pm 18 \%$ for the chest press, $40 \pm 23.6 \%$ for the leg press, $50 \pm 27 \%$ for the upper back, and $38 \pm 31 \%$ for the quadriceps extension (all $P<0.0001$ versus baseline; see Fig. 1). The increase in strength in men was larger than that in women for all four machines $(P<0.05)$, but when the change in strength was expressed as a percentage of baseline strength between the two sexes, this difference was not significant for any exercise $(P>$ 0.26). Men and women thus responded to resistance training similarly in terms of strength change.

After 8 weeks of training, there was an increase in LBM of $1.75 \pm 1.94 \mathrm{~kg}$ (mean $\pm \mathrm{SD}, P<0.0002$ versus baseline) in the entire study group, with a concomitant decline in fat of $0.92 \pm 2.22 \mathrm{~kg}(P<$ 0.05 versus baseline; see Fig. 2). Bone mineral content did not change significantly $(-9.8 \pm 128 \mathrm{~g}, P$ $<0.7)$, nor did weight $(+0.82 \pm 2.45 \mathrm{~kg}, P<0.12)$. There were no significant differences in these changes between men and women, between patients receiving highly active antiretroviral therapy and those not, between those ingesting more than $30 \mathrm{kcal} / \mathrm{kg}$ energy per day $(\mathrm{n}=15)$ and those not, or between those ingesting more than $1 \mathrm{~g} / \mathrm{kg}$ protein per day $(\mathrm{n}=14)$ and those not. Furthermore, there was no correlation between the amount of lean gained and starting viral load, dietary energy intake, or dietary protein intake. 


\section{Change in strength and body composition after 8 weeks of self-selected activity}

At the end of the PRT phase, subjects were asked to return to their usual activity pattern. The goal of this phase was to examine the change in strength and body composition during a period of time without investigator-initiated intervention, in order to test the hypothesis that gains in strength and body composition obtained during the first phase of the study would persist for an additional 8 weeks without further intervention. No dietary advice was given during the second phase of the study beyond what all participants received as part of their participation in the larger cohort study (eat a balanced diet, including adequate calories for weight maintenance and approximately 1.2 $\mathrm{g} / \mathrm{kg}$ protein). Therefore, during this phase subjects were not trained at our center, nor were they in contact with their trainers or dietitians from the first phase. At the end of 8 weeks of this phase, 21 of the 25 subjects returned to the GCRC for a repeat evaluation of their strength and body composition (see Methods). As shown in Fig. 1, the improved strength seen after 8 weeks of PRT was still present at week 16, after 8 weeks of self-selected activity, for the leg press $(P<0.0001$ versus baseline), chest press $(P<0.0003$ versus baseline), upper back $(P<0.0001$ versus baseline), and quadriceps $(P<0.0005$ versus baseline). Compared with week 8 , only the upper back exercise showed a significant decline in strength at week 16 (of $12.0 \mathrm{~kg}, \mathrm{P}<0.0002$ ), whereas the 1 -RMs for the other three exercises were not significantly reduced between weeks 8 and 16 .

At week 16, the mean increase in lean mass in those who completed the entire study was $1.40 \pm 1.8$ $\mathrm{kg}(P<0.003)$. The change in fat mass was, however, no longer significant $(1.05 \pm 3.3 \mathrm{~kg}, P<0.18$, Fig. 2). Weight remained unchanged $(-0.06 \pm 4.1 \mathrm{~kg}, P=0.95)$, as did bone mineral content $(-16 \pm$ $114, P=0.5)$. There was no difference in the gains at week 16 between the men and the women, between black and white subjects, or between the two patients whose medication regimen changed and the rest of the group.

\section{Effect of continued individual training during phase 2}

Nine of the subjects continued to lift weights at least once per week (mean $0.9 \pm 0.4$ sessions per week) on their own at a nearby gym or health club, whereas the other 12 subjects performed no further resistance training. Those who continued to train had a higher mean energy intake (2956 \pm 709 versus $2237 \pm 577 \mathrm{kcal} /$ day, $P<0.03)$ and protein intake $(117 \pm 40$ versus $81 \pm 22.4 \mathrm{~g} / \mathrm{day}, P<$ 0.04 ) at week 16 than those who stopped training, although their intakes had not differed at week 8. Because weights did not change during the second phase despite increased dietary intake, it is reasonable to conclude that the physical activity level of the subjects who continued to train was therefore higher than those who stopped training, so that both groups remained in energy balance. There were no significant differences between these two subgroups in terms of their mean viral load, CD4 counts, or medication use (data not shown). Compared with subjects who did not train, those who did tended to have greater strength at week 16 in the leg press $(P<0.06)$ and chest press $(P<0.07)$, but not on the leg extension or upper back exercises $(P<0.4$ for both). Nonetheless, among those who continued to train to some extent, lean mass increased by a mean of $1.1 \pm 1.6 \mathrm{~kg}$ over this 8 week period $(P<0.05$ versus baseline), whereas those who did no further training showed no change in lean mass $(+0.23 \pm 1.4 \mathrm{~kg}, P=$ NS versus baseline). The difference between the 
two groups was not, however, significant $(P<0.2)$. The group who continued to exercise showed a decline in fat mass of $-0.15 \pm 1.8$ versus $-0.15 \pm 1.7 \mathrm{~kg}$ in the non-exercising group $(P=0.95)$.

\section{Effect of progressive resistance training in wasted subjects}

Patients who met the criteria for AIDS wasting had a somewhat different response to PRT than that seen in the weight-stable patients (Fig. 3). All six subjects with wasting completed the entire protocol. There was a tendency for lean mass in the wasted patients to increase more after 8 weeks of PRT compared with the rest of the group (2.8 versus $1.4 \mathrm{~kg}, P<0.08$ ), and this trend persisted at week 16 (2.4 versus $1.1 \mathrm{~kg}, P<0.09$ ). When the results of the non-wasted subjects were analysed excluding the wasted patients, the increase in lean mass remained significant $(1.4 \mathrm{~kg}, P<0.003)$.

Fat mass increased in the wasted patients at week 8, whereas it fell in the rest of the subjects (+ 0.95 versus $-1.5 \mathrm{~kg}, P<0.002)$. This effect persisted at week $16(+1.6$ versus $-1.9 \mathrm{~kg}, P<0.01)$. As a result, weight gain in the wasted patients was significantly greater than in the rest of the group at both week 8 ( +3.8 versus $-0.2 \mathrm{~kg}, P<0.002$ ) and week 16 ( +4.0 versus in $-1.6 \mathrm{~kg}, P<0.0002$ ). In addition, dietary energy (41.2 versus $32.6 \mathrm{kcal} / \mathrm{kg}, P<0.12$ ) and protein intake (1.65 versus 1.20 $\mathrm{g} / \mathrm{kg}, P<0.11$ ) tended to be higher in the wasted patients than in the non-wasted patients, perhaps indicating a greater level of appetite stimulation by PRT in the wasted group.

\section{Discussion}

The results of this study suggest that a short period of intensive PRT is efficacious in increasing strength and LBM, and reducing fat mass, in adults with HIV infection. The intensity of the intervention was maintained by repeating 1-RM measurements every 2 weeks, and by increasing the resistance whenever subjects' self-reported relative perceived exertion dropped below 16 out of 20 points on a Borg scale [22].

In addition, these data indicate for the first time that the gain in strength and lean mass in HIVinfected individuals is maintained during a short-term follow-up without further directed PRT. This gain was maintained even in subjects who ceased training after the 8 week intervention period, although not surprisingly it appeared that those subjects who elected to continue some form of PRT on their own had continued increases in lean mass, whereas those who stopped training maintained, but did not increase, their LBM. Continued training during the second phase of the study was associated with higher caloric and protein intake, suggesting that the further increase in LBM seen in this subgroup may have been caused by the effect of PRT on dietary intake, an effect previously demonstrated in frail elderly nursing home patients [18]. The mixed results seen in terms of strength change during the second 8 weeks of the study suggest that subjects reduced the intensity, quantity, or type of workouts in the absence of a trainer, so that they maintained their strength to a greater extent than the individuals who ceased training, but they did not continue to gain strength at the same rate seen during the first 8 weeks of the study.

The magnitude of the increase in LBM seen in this study was similar to the $3 \mathrm{~kg}$ increase in lean mass and $1.7 \mathrm{~kg}$ decrease in fat mass shown by Schambelan et al. [12] with 12 weeks of recombinant human growth hormone treatment in patients with AIDS wasting, especially if their results are compared with those seen in the subgroup of wasted patients in the current study. The 
current results are also similar to those seen by Engelson et al. [14] with 12 weeks of testosterone cypionate, which caused a $1.2 \mathrm{~kg}$ increase in lean mass and a $0.2 \mathrm{~kg}$ decrease in fat, estimated by bioelectrical impedance. Gold et al. [13] examined LBM after 16 weeks of nandrolone decanoate in an open-label study in 24 patients with HIV infection, of whom 17 completed the study. They found an increase of $3 \mathrm{~kg}$ in lean mass without a change in fat mass by anthropometry, but no significant change in either compartment by bioelectrical impedance. In contrast, 12 weeks of megestrol acetate increased lean mass by $1.1 \mathrm{~kg}$, and fat mass by approximately $6 \mathrm{~kg}$ [11].

Only two previous published studies $[23,24]$ of strength training have been reported in HIVinfected men. Rigsby et al. [24] examined the effect of combined aerobic and resistance training on strength and aerobic fitness in 37 seropositive men. They found an increase in strength but did not report changes in body composition. The only study to report body composition changes [23] used a moderately intensive protocol, early in the HIV epidemic in 24 men who had recently recovered from Pneumocystis carinii infection. They were all taking AZT, and were randomly assigned to training or control (usual activity) intervention for 6 weeks. The subjects in the intervention group were trained three times a week using a hydraulic isokinetic resistance training machine to train the knee, shoulders, and chest in extension and flexion. There were significant improvements of approximately $20 \%$ in power and torque production in the knee and shoulder with training, compared with moderate deteriorations of approximately $10 \%$ in the control group. In addition, there was a mean weight loss of $1.9 \mathrm{~kg}$ in the control group compared with a gain of $1.7 \mathrm{~kg}$ in the training group $(P<0.0001)$, and a significant improvement in combined mid-arm and mid-thigh circumference in the training group compared with a decline in the control group $(P<0.003)$. These data, although encouraging, are difficult to interpret in the modern era of highly active antiretroviral therapy, for several reasons. First, the study is limited to men, so its relevance to women is unclear. Second, these men were recovering from

P. carinii pneumonia (PCP) at a time (1987-1988) when this condition, although treatable, was often associated with poor long-term survival, and the control subjects lost weight and strength during the study. Third, the body composition response to the training program was measured anthropometrically, which may have limited its precision. Finally, there was no follow-up after the exercise intervention to examine the durability of the effect.

In contrast, the patients in the current study were clinically stable, and were not suffering from opportunistic infections or other acute illnesses. AIDS wasting was not required for entry into this study, since it is likely that improved strength and lean mass will be beneficial to all patients with HIV, and may be more useful as a preventive than a therapeutic measure, to increase body protein stores before acute illness occurs. Unlike other studies $[24,25]$ of aerobic or aerobic plus moderate resistance training in HIV-infected adults, which reported dropout rates of 20 and $76 \%$, we had a very low dropout rate during the exercise study (one out of 25 subjects, or 4\%), and only a slightly higher rate during the follow-up period (three out of 24 subjects, or 12.5\%), despite a high proportion of homeless participants. There were no injuries associated with the PRT protocol, and the subjects were generally adherent to the 3 day per week schedule. A surprising result of this study is that PRT caused both lean and fat gain in wasted subjects, whereas the patients without clinical wasting experienced a gain in lean and a loss of fat, as has been reported in studies of non- 
HIV-infected adults [26]. We chose to study untrained patients without requiring wasting as a criterion of entry to the study, because increasing protein stores and strength by increasing muscle should be of benefit to patients with HIV, regardless of whether or not they have wasting. The results seen in this study are advantageous to both groups, as the wasted patients benefit from increases in both fat and lean mass, whereas the heavier subjects benefit from reducing their fat mass as well as from increasing their lean mass. To our knowledge this is the first demonstration of such an effect by any anabolic intervention.

There are several limitations of this study that should be considered. First, there was no control group, largely because when the study was first designed in 1993, we could not recruit subjects for a randomized study. We are now recruiting patients with evidence of unintentional weight loss or a low BMI for such a controlled trial. In a previous study [19] of healthy HIV-negative individuals, using the same training methods, equipment, assessment techniques, and DXA methods, we found that 24 sessions of sham exercise over 12 weeks, consisting of stretching and swimming for $30 \mathrm{~min}$, did not alter strength (mean change in $1-\mathrm{RM}, 9 \pm 8 \%)$ or LBM $(-0.8 \pm 1.0 \mathrm{~kg})$. In addition, there is a wealth of information indicating that spontaneous increases in LBM, our primary outcome, do not occur even in healthy weight-stable adults over time [27], and are not expected to occur in HIVinfected adults. Rather, the opposite is true: a decline in muscle mass occurs with normal ageing [27], and is accelerated in HIV infection [1,5]. A before-after study design, which implicitly assumes a comparison of the results seen to a null hypothesis of no change, thus seems reasonable to test the efficacy of PRT. Furthermore, the post-exercise ad libitum activity phase of the study offered an opportunity to assess the durability of the increase in LBM achieved during the first 8 weeks: a further increase in lean mass was seen only in the subjects who continued to train, whereas no further increase was seen in the subjects who stopped training.

Another limitation is the short duration of the intervention period. When this study was first submitted for funding in 1993, there was concern about the duration of intervention that could be done without a change in medications that might alter weight and thus lean and fat mass. Although medication stability for a longer duration is now easier to achieve, eliminating this potentially significant confounder was sufficiently important to warrant a short study. On the other hand, this should not be taken to suggest that a longer period of PRT would not have a larger effect on lean mass and strength, as shown by the subgroup of patients who chose to continue to train on their own during the second phase of the study. In previous studies [28], continued improvement in strength and body composition without a plateau effect was seen for up to a year in elderly women. The 8 week PRT phase of this study may, however, be considered a model for an intensive rehabilitative approach to repleting lean mass and strength in HIV-infected individuals, which could be followed in clinical practice by a maintenance phase of less intensive exercise. The demonstration of the efficacy of PRT in the current study, if replicated in larger trials, may be helpful in convincing third party payers to reimburse for an 8 week intensive PRT treatment in the future.

Data from this study are consistent with those seen in other strength training studies [26], which suggest that in order to cause muscle hypertrophy and increase LBM, weight-lifting must be intensive, progressive, and must be performed regularly. Most untrained persons will not 
spontaneously exercise at such an intensive level, nor will they increase the weight without prompting. In this, the role of the trainer is crucial in providing encouragement, promoting good form, and assisting with both the intensiveness and progressiveness of the regimen, at least initially. Studies or clinical applications of PRT, which do not include safeguards to ensure that a high level of resistance training is maintained, are unlikely to be successful. The willingness of third party payers to reimburse for training will be a major determinant of the applicability of PRT to the HIV-infected population.

In conclusion, this study indicates that PRT has the potential to increase strength and lean mass in patients with HIV infection. Perhaps of equal importance in the age of highly active antiretroviral therapy and the increasing prevalence of abdominal obesity and fat redistribution [29,30], PRT can also reduce fat mass. Moreover, these improvements were maintained over a short-term follow-up, and a substantial proportion of patients who had been taught to perform PRT continued to do so on their own after the training phase of the study ended. These data indicate that PRT offers an alternative to pharmacological anabolic treatment to build LBM in HIV infection. Direct comparisons of PRT with other anabolic treatments are now in progress, and consideration should be given to how to incorporate PRT into clinical care in HIV infection.

\section{Acknowledgements}

We wish to thank James Raymond, Julianne Hamel, Elizabeth Tiemann and the staff of Tufts Nutrition for Life for their assistance with subject recruitment; Tracey DiRusso, RPT, and Mary Curran, RPT, for their assistance with training; Barbara Tanenbaum, MS,

RD, for her assistance with dietary record collection; and James Fauntleroy and Abby Shevitz, MD, $\mathrm{MPH}$, for their assistance with data management. 


\section{References}

1. Kotler DP, Tierney AR, Pierson RN: Magnitude of body cell mass depletion and the timing of death from wasting in AIDS. Am J Clin Nutr 1989, 50:444-447.

2. Nahlen BL, Chu SY, Nwanyanwu OC, Berkelman RL, Martinez SA, Rullan JV: HIV wasting syndrome in the United States. AIDS 1993, 7:183-188.

3. Suttmann U, Ockenga J, Sleberg O, Hoogestraat L, Deicher H, Muller MJ: Incidence and prognostic value of malnutrition and wasting in human immunodeficiency virus-infected outpatients. $J$ Acquir Immune Defic Syndr Hum Retrovirol 1995, 8:239-246.

4. Grunfeld C, Feingold KR: Metabolic disturbances and wasting in the acquired immunodeficiency syndrome. N Engl J Med 1992, 327:329-337.

5. Macallan DC, Noble C, Baldwin C, et al.: Energy expenditure and wasting in human immunodeficiency virus infection. N Engl J Med 1995, 333:83-88.

6. Gorbach SL, Knox TA, Roubenoff R: Interactions between nutrition and infection with human immunodeficiency virus. Nutr Rev 1993, 51:226-234.

7. Roubenoff R: Inflammatory and hormonal mediators of cachexia. J Nutr 1997, 127 (Suppl.):1014S-1016.

8. Roubenoff R, Heymsfield SB, Kehayias JJ, Cannon JG, Rosenberg IH: Standardization of nomenclature of body composition in weight loss. Am J Clin Nutr 1997, 66:192-196.

9. Force G, Jockey C, Tugler MH, Khayat G, Chapmetier De Ribes, D: Characteristics of change in body composition with efficiency of antiretroviral treatment in AIDS patients. Nutrition 1997, 13:290.

10. Silva M, Skolnik PR, Gorbach SL, et al:: The effect of protease inhibitors on weight and body composition in HIV-infected patients. AIDS 1998, 12:1645-1651.

11. Von Roenn JH, Armstrong D, Kotler DP, et al.: Megestrol acetate in patients with AIDS-related cachexia. Ann Intern Med 1994, 121:393-399.

12. Schambelan M, Mulligan K, Grunfeld C, et al.: Recombinant human growth hormone in patients with HIV-associated wasting. Ann Intern Med 1996, 125: 873-882.

13. Gold J, High HA, Li Y, et al:: Safety and efficacy of nandrolone decanoate for treatment of wasting in patients with HIV infection. AIDS 1996, 10:745-752.

14. Engelson ES, Rabkin JG, Rabkin R, Kotler DP: Effects of testosterone upon body composition. J Acquir Immune Defic Syndr Hum Retrovirol 1996, 11:510-511.

15. Felig P, Baxter JD, Frohman LA: Endocrinology and Metabolism, 3rd edn. New York: McGraw-Hill; 1995.

16. Gann PH, Hennekens CH, Ma J, Longcope C, Stampfer MJ: Prospective study of sex hormone levels and risk of prostatic cancer. J Natl Cancer Inst 1996, 88:1118-1126.

17. Fiatarone MA, Marks EC, Ryan ND, Meredith CN, Lipsitz LA, Evans WJ: High-intensity strength training in nonagenarians. Effects on skeletal muscle. JAMA 1990, 263:3029-3034.

18. Fiatarone MA, O'Neill EF, Ryan ND, et al.: Exercise training and nutritional supplementation for physical frailty in very elderly people. $N$ Engl J Med 1994, 330:1769-1775.

19. Rall LC, Meydani SN, Kehayias JJ, Dawson-Hughes B, Roubenoff, R: The effect of progressive resistance training in rheumatoid arthritis: increased strength without changes in energy balance or body composition. Arthritis Rheum 1996, 39:415-426. 
20. Roubenoff R, Rall LC, Veldhuis JD, et al:: The relationship between growth hormone kinetics and sarcopenia in postmenopausal women: the role of fat mass and leptin. J Clin Endocrinol Metab 1998, 83:1502-1506.

21. Economos CD, Nelson ME, Fiatarone MA, et al.: A multi-center comparison of dual energy X-ray absorptiometers: in vivo and in vitro soft tissue measurement. Eur J Clin Nutr 1997, 51:312-317.

22. Borg G, Linderholm H: Exercise performance and perceived exertion in patients with coronary insufficiency, arterial hypertension, and vasoregulatory asthenia. Acta Med Scand 1970, 187:17-26.

23. Spence DW, Galantino MLA, Mossberg KA, Zimmerman SO: Progressive resistance exercise: effect on muscle function and anthropometry of a select AIDS population. Arch Phys Med Rehabil 1990, 71:644-648.

24. Rigsby LW, Dishman RK, Jackson AW, MacLean GS, Raven PB: Effects of exercise training on men seropositive for the human immunodeficiency virus-1. Med Sci Sports Exerc 1992, 24:6-12.

25. MacArthur RD, Levine SD, Birk TJ: Supervised exercise training improves cardiopulmonary fitness in HIV-infected persons. Med Sci Sports Exerc 1993, 25:684688.

26. Baechle TR: Essentials of Strength Training and Conditioning. Champaign, IL: Human Kinetics; 1994.

27. Kehayias JJ, Fiatarone MF, Zhuang H, Roubenoff R: Total body potassium and body fat: relevance to aging. Am J Clin Nutr 1997, 66:904-910.

28. Morganti CM, Nelson ME, Fiatarone MA, Dallal GE, Economos CD, Crawford BM, Evans WJ: Strength improvements with 1 year of progressive resistance training in older women. Med Sci Sports Exerc 1995, 27:906-912.

29. Miller KD, Jones E, Yanovski JA, Shankar R, Feuerstein I, Falloon, J: Visceral abdominalfat accumulation associated with use of indinavir. Lancet 1998, 351:871-875.

30. Lo JC, Mulligan K, Tai VW, Algren H, Schambelan M: 'Buffalo hump' in men with HIV-1 infection. Lancet 1998, 351:867-870. 


\section{Tables \& Figures}

Table 1: Demographic and Clinical Characteristics of the Study Population

\begin{tabular}{|c|c|c|c|}
\hline Parameter & Baseline & Week 8 & Week 16 \\
\hline Male:female & $20: 5$ & 19:5 & $16: 5$ \\
\hline Age & $38.8(7.8)$ & - & - \\
\hline Ethnicity - white: African-American & $14: 11$ & $14: 10$ & $12: 9$ \\
\hline \multicolumn{4}{|l|}{ HIV risk factor } \\
\hline Intravenous drug use & 9 & 8 & 4 \\
\hline Homosexual & 15 & 15 & 15 \\
\hline Transfusion & 1 & 1 & 1 \\
\hline \multicolumn{4}{|l|}{ Antiretroviral therapy } \\
\hline None & 2 & 2 & 2 \\
\hline Monotherapy/dual therapy & 9 & 8 & 7 \\
\hline HAART & 14 & 14 & 12 \\
\hline CD4 count/ml & $328(151)$ & 303 (189) & $357(172)$ \\
\hline Circulating HIV RNA, copies/ml & 8919 (18 408) & $9272(22386)$ & 8752 (20 998) \\
\hline Hemoglobin, g/dl & $14.0(1.3)$ & $14.0(1.2)$ & $13.8(1.3)$ \\
\hline \multicolumn{4}{|l|}{ Dietary intake } \\
\hline Energy, kcal/day & $2590(723)$ & $2604(778)$ & $2456(682)$ \\
\hline Protein, g/day & $97.3(34.7)$ & $102.7(38.6)$ & $99.6(33.9)$ \\
\hline \multicolumn{4}{|l|}{ Body composition } \\
\hline Weight, kg & $76.0(14.1)$ & 76.9 (13.9) & $77.8(14.1)$ \\
\hline Lean body mass, kg & $53.1(7.7)$ & $54.9(8.9)$ & $55.1(8.8)$ \\
\hline Fat mass, kg & $20.3(11.7)$ & $19.4(11.0)$ & $20.3(12.7)$ \\
\hline Bone mineral content, $\mathrm{kg}$ & $2.6(0.4)$ & $2.6(0.4)$ & $2.6(0.4)$ \\
\hline
\end{tabular}

Data are mean (SD) unless otherwise indicated. HAART, Highly active antiretroviral therapy.

Compartment weights may not add up to total weight due to rounding. 
Figure 1

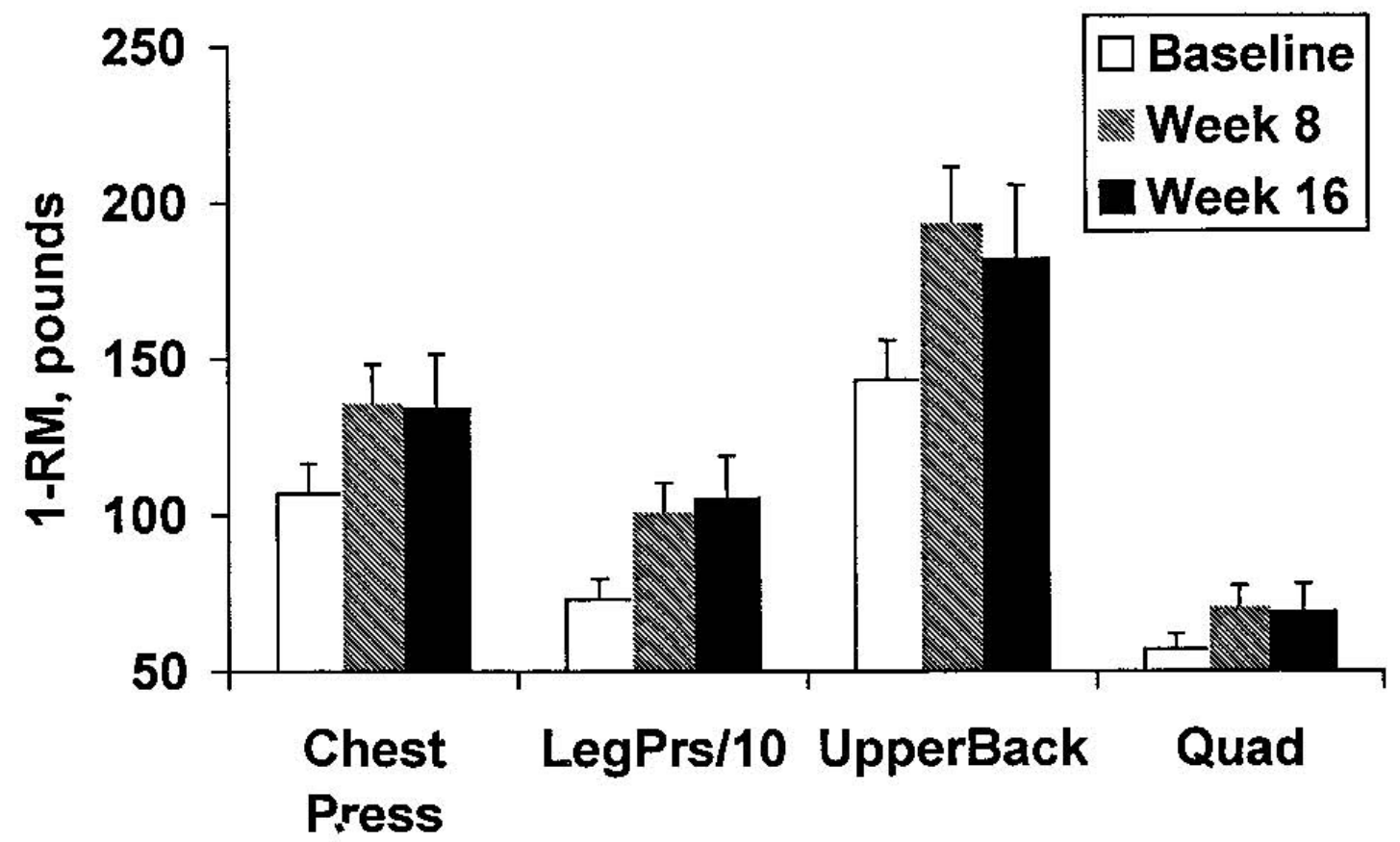

One-repetition maximum strength (lb) at baseline, after 8 weeks of PRT, and after an additional 8 weeks of ad libitum activity for each of the four exercise machines used in the study. Note that the leg press results are shown divided by 10 in order to fit on the same scale as the other machines. All changes are significant $(P<0.0001)$ versus baseline at both 8 and 16 weeks. See text for statistical details. 
Figure 2

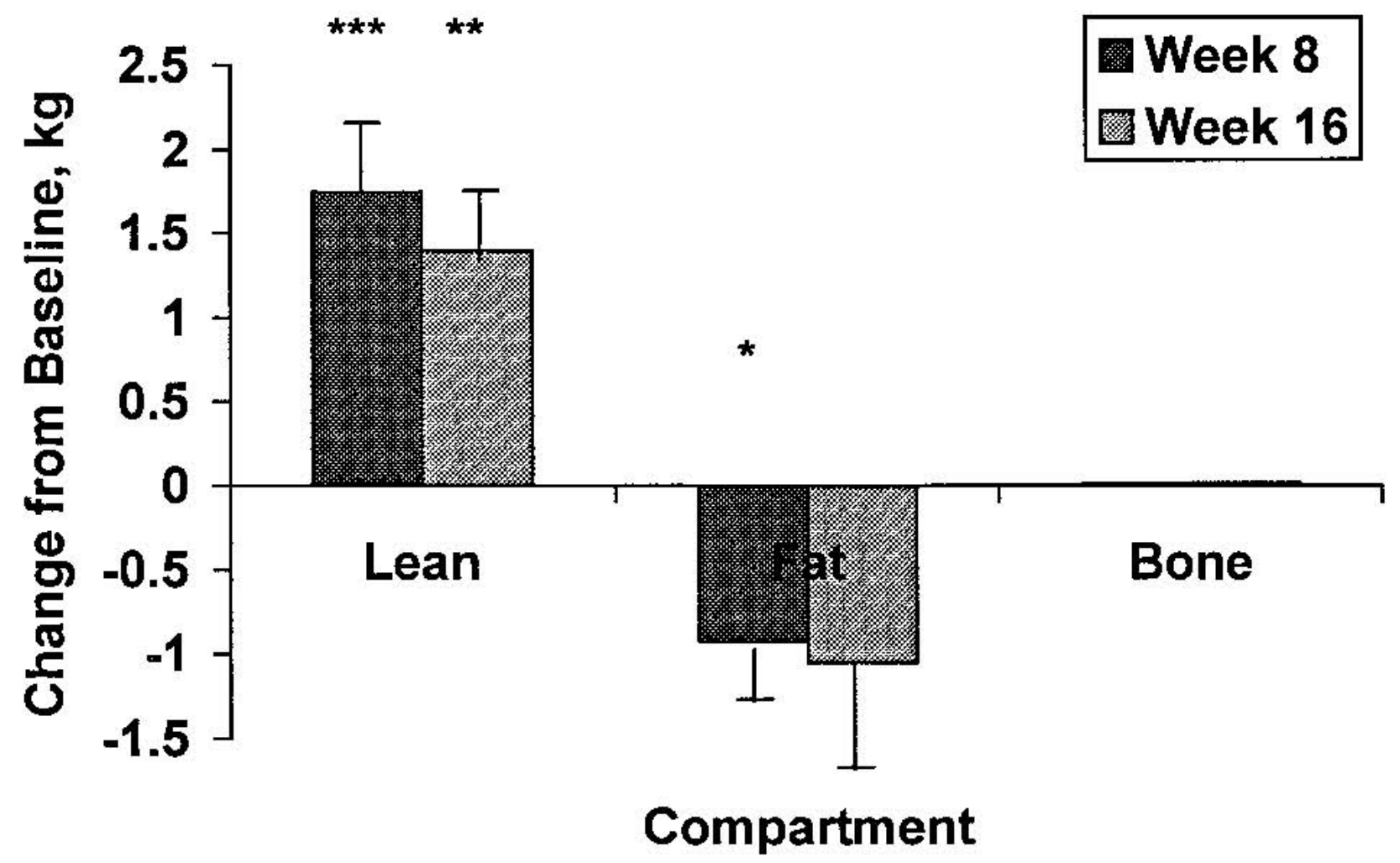

Change from baseline in LBM, fat mass and bone mass at the end of the 8 week PRT phase of the study and at the end of the 16 week study period. ${ }^{*} P<0.05$; ${ }^{* *} P<0.003$; ${ }^{* * *} P<0.0002$, all versus baseline. 
Figure 3

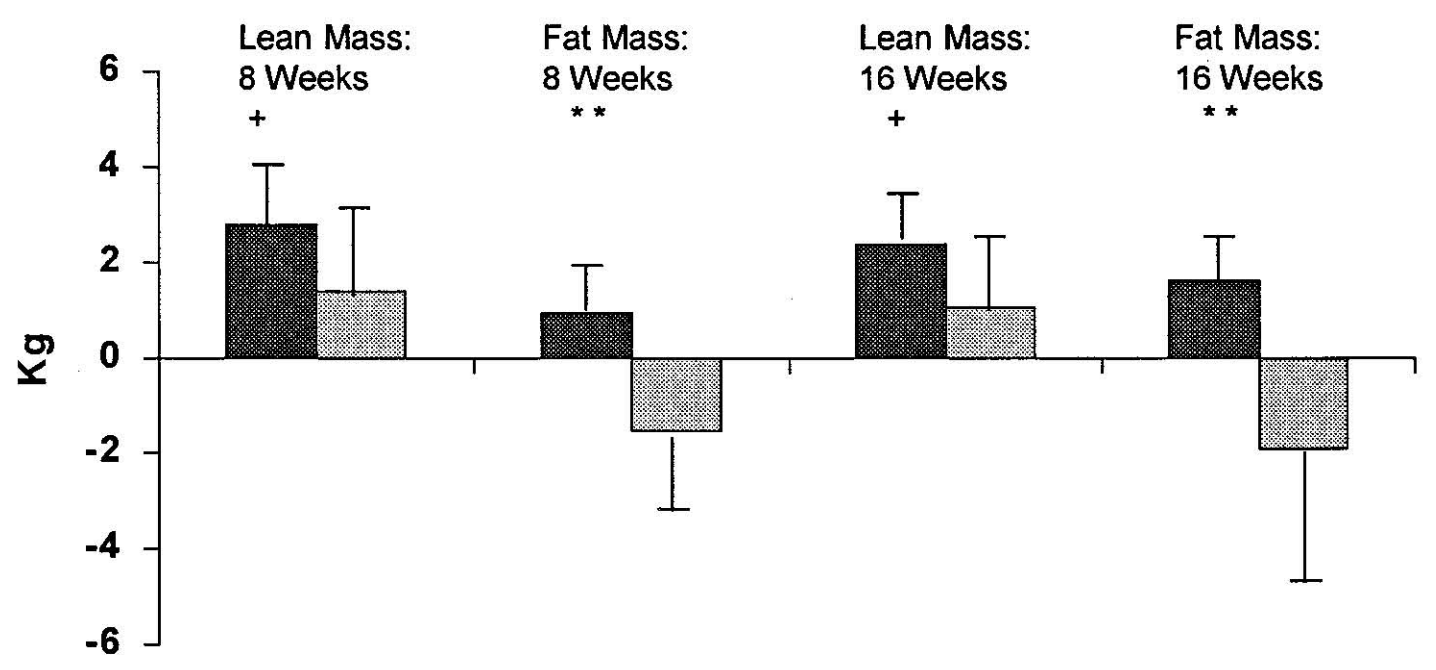

Wasted Not Wasted

Change from baseline in LBM and fat mass at the end of 8 weeks of PRT and at the end of the 16 week study period, in subjects with or without AIDS wasting. ${ }^{\dagger} P<0.09$; ${ }^{* *} P<0.002$ 